ESAIM: COCV 19 (2013) 63-77

DOI: $10.1051 / \mathrm{cocv} / 2011200$
ESAIM: Control, Optimisation and Calculus of Variations

www.esaim-cocv.org

\title{
DETERMINISTIC MINIMAX IMPULSE CONTROL IN FINITE HORIZON: THE VISCOSITY SOLUTION APPROACH *,**
}

\author{
BRAHIM EL ASRI ${ }^{1}$
}

\begin{abstract}
We study here the impulse control minimax problem. We allow the cost functionals and dynamics to be unbounded and hence the value functions can possibly be unbounded. We prove that the value function of the problem is continuous. Moreover, the value function is characterized as the unique viscosity solution of an Isaacs quasi-variational inequality. This problem is in relation with an application in mathematical finance.
\end{abstract}

Mathematics Subject Classification. 34H05, 34K35, 49L20, 49L25.

Received July 13, 2010. Revised October 13, 2010.

Published online March 22, 2012.

\section{INTRODUCTION}

In this paper we study an optimal impulse control problem with finite horizon.

Optimal impulse control problems appear in many practical situations. We refer the reader to [3] (and the references cited therein) for extensive discussions. For deterministic autonomous systems with infinite horizon, optimal impulse control problems were studied in [1], and optimal control problems with continuous, switching, and impulse controls were studied by the author [17] (see also [18]). Differential games with switching strategies in finite and infinite duration were also studied [19,20]. Yong, in [9], also studies differential games where one person uses an impulse control and other uses continuous controls. Recently El Farouq et al. [10] extended the work of Yong [21] in the finite horizon case but allowing general jumps. In all these works the existence of the value functions of optimal impulse control problem and uniqueness of viscosity solution are obtained assuming that the dynamics and costs functionals are bounded and the impulse cost function should not depend on $y$.

Our aim in this work is to relax the boundedness assumption on cost functionals and the dynamics for impulse control problem and the impulse cost function should depend on $x$. Therefore the main objective of our work, and this is the novelty of the paper, is to characterize the value function as the only solution in viscosity sense of the associated quasivariational inequality for the finite horizon problem in suitable subclass of bounded

\footnotetext{
Keywords and phrases. Impulse control, robust control, differential games, quasi-variational inequality, viscosity solution.

* The research leading to these results has received funding in part by the European Community's FP 7 Programme under contract agreement PITN-GA-2008-213841, Marie Curie ITN "Controlled Systems".

** A significant part of this work was done when the author was affiliated to Le Mans University.

1 Institut für Stochastik, Friedrich-Schiller-Universität Jena, Ernst-Abbe-Platz 2, 07743 Jena, Germany.

brahim.el-asri@uni-jena.de
} 
from below continuous functions, with linear growth when the dynamics unbounded and costs functionals are bounded from below with linear growth and the impulse cost function depends on $x$.

This paper is organized as follows:

In Section 2, we formulate the problem and we give the related definitions. In Section 3, we give some properties of the value function, especially the dynamic programming principle. Further we provide some estimates for the optimal strategy of the optimal impulse control problem which, in combination with the dynamic programming principle, play a crucial role in the proof of the existence of the value function. Section 4 is devoted to the connection between the optimal impulse control problem and quasi-variational inequality. In Section 5, we show that the solution of QVIs is unique in the subclass of bounded from below continuous functions which satisfy a linear growth condition.

\section{Formulation of the Problem AND PRELiminary ReSUlts}

\subsection{Setting of the problem}

Let a two-players differential game system be defined by the solution of following dynamical equations

$$
\left\{\begin{array}{l}
\dot{y}(t)=f(t, y(t), \tau(t)) \\
y\left(t_{0}\right)=x \in \mathbb{R}^{m} \\
y\left(t_{k}^{+}\right)=y\left(t_{k}^{-}\right)+g\left(t_{k}, y\left(t_{k}^{-}\right), \xi_{k}\right), t_{k} \geq t_{0}, \xi_{k} \neq 0
\end{array}\right.
$$

where $y(t)$ is the state of the system, with values in $\mathbb{R}^{m}$, at time $t, x$ is the initial state. The time variable $t$ belongs to $\left[t_{0}, T\right]$ where $0 \leq t_{0}<T$, and $y\left(t_{k}^{ \pm}\right)=\lim _{t \rightarrow t_{k}^{ \pm}} y(t)$. We assume that $y$ is left continuous at the times $t_{k}$ : $y\left(t_{k}^{-}\right)=y\left(t_{k}\right), k \geq 1$.

The system is driven by two controls, a continuous control $\tau(t) \in \mathcal{K} \in \mathbb{R}^{m}$, where $\mathcal{K}$ is compact set, and an impulsive control defined by a double sequence $t_{1}, \ldots, t_{k}, \ldots, \xi_{1}, \ldots, \xi_{k}, \ldots$,

$k \in \mathbb{N}^{*}=\mathbb{N} \backslash\{0\}$, where $t_{k}$ are the strategy, $t_{k} \leq t_{k+1}$ and $\xi_{k} \in \mathbb{R}^{m}$ the control at time $t_{k}$ of the jumps in $y\left(t_{k}\right)$. Let $\mathcal{S}:=\left(\left(t_{k}\right)_{k \geq 1},\left(\xi_{k}\right)_{k \geq 1}\right)$ the set of these strategies denoted by $\mathcal{D}$.

For any initial condition $\left(t_{0}, x\right)$, controls $\tau(\cdot)$ and $\mathcal{S}$ generate a trajectory $y(\cdot)$ of this system. The pay-off is given by the following:

$$
J\left(t_{0}, x, \mathcal{S}, \tau(.)\right)=\int_{t_{0}}^{T} \psi(s, y(s), \tau(s)) \mathrm{d} s+\sum_{k \geq 1} C\left(t_{k}, y\left(t_{k}\right), \xi_{k}\right) \mathbb{1}_{\left[t_{k} \leq T\right]}+G(y(T)),
$$

where if $t_{k}=T$ for some $k$ then we take $G(y(T))=G\left(y\left(T^{+}\right)\right)$. The term $C\left(t_{k}, y\left(t_{k}\right), \xi_{k}\right)$ is called the impulse cost. It is the cost when player- $\xi$ makes an impulse $\xi_{k}$ at time $t_{k}$. In the game, player- $\xi$ would like to minimize the pay-off (2.2) by choosing suitable impulse control $\xi($.$) , whereas player- \tau$ wants to maximize the pay-off $(2.2)$ by choosing a proper control

$$
\tau(.) \in \Omega=\left\{\text { measurable functions }\left[t_{0}, T\right] \rightarrow \mathcal{K}\right\} .
$$

We shall sometimes write $\tau \in \Omega$ instead of $\tau(.) \in \Omega$.

We now define the admissible strategies $\varphi$ for the minimizing impulse control $\mathcal{D}$, as non-anticipative strategies. We shall let $\mathcal{D}_{a}$ be the set of all such non-anticipative strategies.

Definition 2.1. A map $\varphi: \Omega \rightarrow \mathcal{S}$ is called a non-anticipative strategy if for any two controls $\tau_{1}($.$) and \tau_{2}($.$) ,$ and any $t \in\left[t_{0}, T\right]$, the condition on their restrictions to $\left[t_{0}, t\left[:\left.\tau_{1}\right|_{\left[t_{0}, t[\right.}=\left.\left.\tau_{2}\right|_{\left[t_{0}, t[\right.} \operatorname{implies} \varphi\left(\tau_{1}\right)\right|_{\left[t_{0}, t\right]}=\left.\varphi\left(\tau_{2}\right)\right|_{\left[t_{0}, t\right]}\right.\right.$.

In the next, we define the value function of the problem $v:[0, T] \times \mathbb{R}^{m} \rightarrow \mathbb{R}$ as

$$
v\left(t_{0}, x\right)=\inf _{\varphi \in \mathcal{D}_{a}} \sup _{\tau(.) \in \Omega} J\left(t_{0}, x, \varphi(\tau(.)), \tau(.)\right) .
$$




\subsection{Assumptions}

Throughout this paper $T$ (resp. $m$ ) is a fixed real (resp. integer) positive constant. Let us now consider the followings:

(1) $f:[0, T] \times \mathbb{R}^{m} \times \mathcal{K} \rightarrow \mathbb{R}^{m}$ and $g:[0, T] \times \mathbb{R}^{m} \times \mathbb{R}^{m} \rightarrow \mathbb{R}^{m}$ are two continuous functions for which there exists a constant $C \geq 0$ such that for any $t \in[0, T], \tau \in \mathcal{K}$ and $\xi, x, x^{\prime} \in \mathbb{R}^{m}$

$$
\begin{aligned}
& |f(t, x, \tau)|+|g(t, x, \xi)| \leq C(1+|x|) \quad \text { and } \\
& \left|g(t, x, \xi)-g\left(t, x^{\prime}, \xi\right)\right|+\left|f(t, x, \tau)-f\left(t, x^{\prime}, \tau\right)\right| \leq C\left|x-x^{\prime}\right| ;
\end{aligned}
$$

(2) $C:[0, T] \times \mathbb{R}^{m} \times \mathbb{R}^{m} \rightarrow \mathbb{R}$, is continuous with respect to $t$ and $\xi$ uniformly in $x$ with linear growth

$$
|C(t, x, \xi)| \leq C(1+|x|), \forall(t, x, \xi) \in[0, T] \times \mathbb{R}^{m} \times \mathbb{R}^{m} .
$$

Moreover we assume that there exists a constant $\alpha>0$ such that for any $(t, x, \xi) \in[0, T] \times \mathbb{R}^{m} \times \mathbb{R}^{m}$,

$$
C(t, x, \xi) \geq \alpha
$$

(3) $\psi:[0, T] \times \mathbb{R}^{m} \times \mathcal{K} \rightarrow \mathbb{R}$ is continuous with respect to $t$ and $\tau$ uniformly in $x$ with linear growth,

$$
|\psi(t, x, \tau)| \leq C(1+|x|), \forall(t, x, \tau) \in[0, T] \times \mathbb{R}^{m} \times \mathcal{K},
$$

and is bounded from below;

(4) $G: \mathbb{R}^{m} \rightarrow \mathbb{R}$ is uniformly continuous with linear growth

$$
|G(x)| \leq C(1+|x|), \forall x \in \mathbb{R}^{m},
$$

and is bounded from below.

These properties of $f$ and $g$ imply in particular that $y(t)_{0 \leq t \leq T}$ solution of the standard DE (2.1) exists and is unique, for any $t \in[0, T]$ and $x \in \mathbb{R}^{m}$.

\subsection{Admissible strategies}

We want to investigate the problem of minimizing $\sup _{\tau \in \Omega} J$ through the impulse control. We mean to allow closed loop strategies for the minimizing control. We remark that, being only interested in the inf sup problem, and not a possible saddle point.

Theorem 2.2. Under the standing assumptions (Sect. 2.2) the value function $v$ is bounded from below with linear growth.

Proof. Consider the particular strategy in $\mathcal{D}_{a}$ is the one where we have no impulse time. In this case $\sum_{k \geq 1} C\left(t_{k}, y\left(t_{k}\right), \xi_{k}\right) \mathbb{1}_{\left[t_{k} \leq T\right]}=0$.

$$
v(t, x) \leq \sup _{\tau \in \Omega}\left[\int_{t}^{T} \psi(s, y(s), \tau(s)) \mathrm{d} s+G(y(T))\right] .
$$

Since $\psi$ and $G$ are linear growth, then

$$
v(t, x) \leq \int_{t}^{T} C(1+|y(s)|) \mathrm{d} s+C(1+|y(T)|) .
$$

Now by using standard estimates from ODE, Gronwall's Lemma and the strategy where we have no impulse time, we can show that

$$
|y(t)| \leq C(1+|x|)
$$


where $C$ is constant of $T$. Hence using this estimate we get

$$
v(t, x) \leq C(1+|x|) .
$$

On the other hand, since the cost $C\left(t_{k}, y\left(t_{k}\right), \xi_{k}\right)$ are non negative functions and since $\psi$ and $G$ are bounded from below, then $v$ is bounded from below.

We are now giving some properties of the admissible strategy.

Proposition 2.3. Let $\varphi$ be a non-anticipative nearly optimal strategy composed of impulse control $(\delta, \xi)=$ $\left(\left(t_{n}\right)_{n \geq 1},\left(\xi_{n}\right)_{n \geq 1}\right)$. Then:

$$
\sum_{k \geq 1} C\left(t_{k}, y\left(t_{k}\right), \xi_{k}\right) \mathbb{1}_{\left[t_{k} \leq T\right]} \leq C(1+|x|) .
$$

There exists a positive constant $C$ which does not depend on $t$ and $x$ such that:

$$
\forall n \geq 1, \mathbb{1}_{\left[t_{n} \leq T\right]} \leq \frac{C(1+|x|)}{n} .
$$

Proof. Recall the characterization of (2.2) that reads as:

$$
v(t, x)=\inf _{\varphi \in \mathcal{D}_{a}} \sup _{\tau(.) \in \Omega}\left[\int_{t}^{T} \psi(s, y(s), \tau(s)) \mathrm{d} s+\sum_{k \geq 1} C\left(t_{k}, y\left(t_{k}\right), \xi_{k}\right) \mathbb{1}_{\left[t_{k} \leq T\right]}+G(y(T))\right] .
$$

Let us choose a non-anticipative nearly optimal strategy $\varphi$ composed of impulse control $(\delta, \xi)=$ $\left(\left(t_{n}\right)_{n \geq 1},\left(\xi_{n}\right)_{n \geq 1}\right)$ such that:

$$
\int_{t}^{T} \psi(s, y(s), \tau(s)) \mathrm{d} s+\sum_{k \geq 1} C\left(t_{k}, y\left(t_{k}\right), \xi_{k}\right) \mathbb{1}_{\left[t_{k} \leq T\right]}+G(y(T)) \leq v(t, x)+1 .
$$

Since $v(t, x) \leq C(1+|x|)$ and since $\psi$ and $G$ are bounded from below then we have

$$
\sum_{k \geq 1} C\left(t_{k}, y\left(t_{k}\right), \xi_{k}\right) \mathbb{1}_{\left[t_{k} \leq T\right]} \leq C(1+|x|) .
$$

Next we show (2.7). Taking into account that $C(t, y, \xi) \geq \alpha>0$ for any $(t, y, \xi) \in\left[t_{0}, T\right] \times \mathbb{R}^{m} \times \mathbb{R}^{m}$ we obtain:

$$
\sum_{k \geq 1} \alpha \mathbb{1}_{\left[t_{k} \leq T\right]} \leq C(1+|x|) .
$$

But for any $k \leq n,\left[t_{n} \leq T\right] \subset\left[t_{k} \leq T\right]$ then:

$$
\alpha n \mathbb{1}_{\left[t_{n} \leq T\right]} \leq C(1+|x|) .
$$

Finally taking into account $\alpha>0$, we obtain the desired result.

It may be to the best advantage of the minimizer to make a jump at some time $t$, immediately followed, at the same time, by another jump, and so on. As any such jump entails a cost not less than $\alpha$, from Proposition 2.3 the number of jumps may be restricted, with no loss of generality, to be less than $\bar{k}=\frac{C}{\alpha}(1+|x|)$. To allow for the possibility of several successive but simultaneous jumps, we proceed as follows. Let

$$
E=\bigcup_{n=1}^{\bar{k}}\left(\mathbb{R}^{m}\right)^{n}
$$


We extend $g$ and $C$ from $\mathbb{R}^{m}$ to $E$ in the natural way: let $\xi \in\left(\mathbb{R}^{m}\right)^{p}$ be a multiple jump of multiplicity $p$. Let $\left(\xi^{1}, \xi^{2}, \ldots, \xi^{p}\right)$ be its components. For a given $y \in \mathbb{R}^{m}$, let $z_{0}=y$, and for $l \in\{1, \ldots, p\}, z_{l}=z_{l-1}+g\left(t, z_{l-1}, \xi^{l}\right)$. Then we set

$$
g(t, y, \xi)=z_{p}-y, \quad C(t, y, \xi)=\sum_{l=1}^{p} C\left(t, z_{l-1}, \xi^{l}\right) .
$$

There is no point in considering the possibility of several successive multiple jumps at the same jump time, since a sequence of simultaneous multiple jumps is a multiple jump.

From now on, when we refer to jumps, it will always be multiple jumps, unless specifically referred to as simple jumps. Simple jumps are the same thing as a multiple jump of multiplicity 1 . And of course, in a control $\left(t_{k}, \xi_{k}\right)$ the $\xi_{k}$ are to be considered as multiple jumps. But the $t_{k}$ 's are always assumed to be distinct.

We also state the following definition:

Definition 2.4. For any function $v:\left[t_{0}, T\right] \times \mathbb{R}^{m} \rightarrow \mathbb{R}$, let the operator $N$ be given by

$$
N[v](t, x)=\inf _{\xi \in E}[v(t, x+g(t, x, \xi))+C(t, x, \xi)] .
$$

\section{The VAlue FunCtion}

\subsection{Dynamic programming principle}

The dynamic programming principle is a well-known property in optimal impulse control. In our optimal control problem, it is formulated as follows:

Theorem 3.1 ([10], Prop. 3.1). The value function v(.,.) satisfies the following optimality principle: for all $t \leq t^{\prime} \in\left[t_{0}, T\left[\right.\right.$ and $x \in \mathbb{R}^{m}$,

$$
v(t, x)=\inf _{\varphi \in \mathcal{D}_{a}} \sup _{\tau \in \Omega}\left[\int_{t}^{t^{\prime}} \psi(s, y(s), \tau(s)) \mathrm{d} s+\sum_{k \geq 1, t_{k}<t^{\prime}} C\left(t_{k}, y\left(t_{k}\right), \xi_{k}\right) \mathbb{1}_{\left[t_{k} \leq T\right]}+\mathbb{1}_{\left[t^{\prime} \leq T\right]} v\left(t^{\prime}, y\left(t^{\prime}\right)\right)\right],
$$

and

$$
v(t, x)=\inf _{\varphi \in \mathcal{D}_{a}} \sup _{\tau \in \Omega}\left[\int_{t}^{t_{n}} \psi(s, y(s), \tau(s)) \mathrm{d} s+\sum_{1 \leq k<n} C\left(t_{k}, y\left(t_{k}\right), \xi_{k}\right) \mathbb{1}_{\left[t_{k} \leq T\right]}+\mathbb{1}_{\left[t_{n} \leq T\right]} v\left(t_{n}, y\left(t_{n}\right)\right)\right],
$$

where $\left(\left(t_{n}\right)_{n \geq 1},\left(\xi_{n}\right)_{n \geq 1}\right)$ be an admissible control.

Proposition 3.2. The value function $v(.,$.$) has the following property:$

for all $t \in\left[t_{0}, T\right]$ and $x \in \mathbb{R}^{m}$,

$$
v(t, x) \leq N[v](t, x) .
$$

Proof. Assume first that for some $x$ and $t$ :

$$
v(t, x)>N[v](t, x) .
$$

Then we have for $t \leq t^{\prime}$ :

$$
\begin{aligned}
& \inf _{\varphi \in \mathcal{D}_{a}} \sup _{\tau \in \Omega}\left[\int_{t}^{t^{\prime}} \psi(s, y(s), \tau(s)) \mathrm{d} s+\sum_{k \geq 1, t_{k}<t^{\prime}} C\left(t_{k}, y\left(t_{k}\right), \xi_{k}\right) \mathbb{1}_{\left[t_{k} \leq T\right]}+\mathbb{1}_{\left[t^{\prime} \leq T\right]} v\left(t^{\prime}, y\left(t^{\prime}\right)\right)\right] \\
& >\inf _{\xi \in E}[v(t, x+g(t, x, \xi))+C(t, x, \xi)] .
\end{aligned}
$$


Among the admissible strategy $\varphi^{\epsilon}$ 's there are those that place a jump at time $t$.

$$
\begin{aligned}
\sup _{\tau \in \Omega} & {\left[\int_{t}^{t^{\prime}} \psi(s, y(s), \tau(s)) \mathrm{d} s+\sum_{k \geq 1, t_{k}<t^{\prime}} C\left(t_{k}, y\left(t_{k}\right), \xi_{k}\right) \mathbb{1}_{\left[t_{k}<T\right]}+\mathbb{1}_{\left[t^{\prime}<T\right]} v\left(t^{\prime}, y\left(t^{\prime}\right)\right)\right] } \\
> & v(t, x+g(t, x, \xi))+C(t, x, \xi)-\epsilon .
\end{aligned}
$$

Now, pick $\tau_{1}$ such that

$$
\begin{aligned}
& \int_{t}^{t^{\prime}} \psi\left(s, y(s), \tau_{1}(s)\right) \mathrm{d} s+\sum_{k \geq 1, t_{k}<t^{\prime}} C\left(t_{k}, y\left(t_{k}\right), \xi_{k}\right) \mathbb{1}_{\left[t_{k} \leq T\right]}+\mathbb{1}_{\left[t^{\prime} \leq T\right]} v\left(t^{\prime}, y\left(t^{\prime}\right)\right)+\epsilon \\
& \quad \geq \sup _{\tau \in \Omega}\left[\int_{t}^{t^{\prime}} \psi(s, y(s), \tau(s)) \mathrm{d} s+\sum_{k \geq 1, t_{k}<t^{\prime}} C\left(t_{k}, y\left(t_{k}\right), \xi_{k}\right) \mathbb{1}_{\left[t_{k} \leq T\right]}+\mathbb{1}_{\left[t^{\prime} \leq T\right]} v\left(t^{\prime}, y\left(t^{\prime}\right)\right)\right],
\end{aligned}
$$

which implies that:

$$
\begin{aligned}
& \int_{t}^{t^{\prime}} \psi\left(s, y(s), \tau_{1}(s)\right) \mathrm{d} s+\sum_{k \geq 1, t_{k}<t^{\prime}} C\left(t_{k}, y\left(t_{k}\right), \xi_{k}\right) \mathbb{1}_{\left[t_{k} \leq T\right]}+\mathbb{1}_{\left[t^{\prime} \leq T\right]} v\left(t^{\prime}, y\left(t^{\prime}\right)\right)+\epsilon \\
& >v(t, x+g(t, x, \xi))+C(t, x, \xi)-\epsilon .
\end{aligned}
$$

Choosing now $t^{\prime}=t$, yields the relation

$$
\epsilon+v(t, x+g(t, x, \xi))>v(t, x+g(t, x, \xi))+C(t, x, \xi)-\epsilon .
$$

By sending $\epsilon \rightarrow 0$, we obtain $C(t, x, \xi)<0$, which is a contradiction.

\subsection{Continuity of value function}

In this section we prove the continuity of the value function. The main result of this section can be stated as follows.

We first present some preliminary results on $y($.$) . Consider \varphi \in \mathcal{D}_{a}$ and $(\tau(),. \varphi(\tau()$.$) , composed of jumps$ instants $t_{1}, t_{2}, \ldots, t_{n}$ in the interval $[t, T]$, with jumps $\xi_{1}, \xi_{2}, \ldots, \xi_{n}$, and let $y_{1}($.$) and y_{2}($.$) be the trajectories$ generated by $\mathcal{D}_{a}$, from $y_{i}(t)=x_{i}, i=1,2$.

Lemma 3.3. There exists a constant $C$ such that for any $s \in[t, T], x_{1}, x_{2} \in \mathbb{R}^{m}$, and $k \in\{1,2 \ldots, n\}$

$$
\left|y_{1}(s)-y_{2}(s)\right| \leq \exp (C(s-t))(1+C)^{n}\left|x_{1}-x_{2}\right|
$$

Proof. By the Lipschitz continuity of $f$ and Gronwall's lemma, we have

$$
\left|y_{1}(s)-y_{2}(s)\right| \leq \exp (C(s-t))(1+C)\left|x_{1}-x_{2}\right|, \quad \forall s \in\left[t, t_{1}\right] .
$$

Next let us show for an impulse time

$$
\left|y_{1}\left(t_{k}^{+}\right)-y_{2}\left(t_{k}^{+}\right)\right| \leq \exp \left(C\left(t_{k}-t\right)\right)(1+C)^{k}\left|x_{1}-x_{2}\right| .
$$

Looking more carefully at the first jump and using the Lipschitz continuity of $g$, we have

$$
\begin{aligned}
\left|y_{1}\left(t_{1}^{+}\right)-y_{2}\left(t_{1}^{+}\right)\right| & =\left|y_{1}\left(t_{1}^{-}\right)+g\left(t_{1}^{-}, y_{1}\left(t_{1}^{-}\right), \xi_{1}\right)-y_{2}\left(t_{1}^{-}\right)-g\left(t_{1}^{-}, y_{2}\left(t_{1}^{-}\right), \xi_{1}\right)\right| \\
& \leq(1+C)\left|y_{1}\left(t_{1}^{-}\right)-y_{2}\left(t_{1}^{-}\right)\right| \\
& \leq \exp \left(C\left(t_{1}-t\right)\right)(1+C)\left|x_{1}-x_{2}\right| .
\end{aligned}
$$


The above assertion is obviously true for $k=1$. Suppose now it holds true at step $k$. Then, at step $k+1$,

$$
\begin{aligned}
\left|y_{1}\left(t_{k+1}^{+}\right)-y_{2}\left(t_{k+1}^{+}\right)\right| & \leq(1+C)\left|y_{1}\left(t_{k+1}^{-}\right)-y_{2}\left(t_{k+1}^{-}\right)\right| \\
& \leq(1+C)\left|y_{1}\left(t_{k}^{+}\right)-y_{2}\left(t_{k}^{+}\right)\right| \exp \left(C\left(t_{k+1}^{-}-t_{k}^{+}\right)\right) \\
& \leq \exp \left(C\left(t_{k+1}-t\right)\right)(1+C)^{k+1}\left|x_{1}-x_{2}\right| .
\end{aligned}
$$

Finally

$$
\left|y_{1}(s)-y_{2}(s)\right| \leq \exp (C(s-t))(1+C)^{n}\left|x_{1}-x_{2}\right|, \quad \forall s \in[0, T]
$$

We are now ready to give the main theorem of this article.

Theorem 3.4. The value function $v:[0, T] \times \mathbb{R}^{m} \rightarrow \mathbb{R}$ is continuous in $t$ and $x$.

Proof. Let us consider $\epsilon>0$ and $\left(t^{\prime}, x^{\prime}\right) \in B((t, x), \epsilon)$ and let us consider the following set of strategies:

$$
\tilde{D}_{a}:=\left\{(\delta, \xi)=\left(\left(t_{n}\right)_{n \geq 1},\left(\xi_{n}\right)_{n \geq 1}\right) \in \mathcal{D}_{a} \text { such that } \forall n \geq 1, \mathbb{1}_{\left[\tau_{n} \leq T\right]} \leq \frac{C(1+(\epsilon+|x|))}{n}\right\} .
$$

From Proposition 2.3, the impulse control of non-anticipative strategy optimal $(\delta, \xi)$ belongs to $\tilde{D}_{a}$.

First let us show that $v$ is upper semi-continuous. Recall the characterization of dynamical programming principle that reads as

$$
\begin{aligned}
v(t, x) & =\inf _{\varphi \in \tilde{D}_{a}} \sup _{\tau \in \Omega}\left[\int_{t}^{t_{n}} \psi(s, y(s), \tau(s)) \mathrm{d} s+\sum_{1 \leq k<n} C\left(t_{k}, y\left(t_{k}\right), \xi_{k}\right) \mathbb{1}_{\left[t_{k} \leq T\right]}+\mathbb{1}_{\left[t_{n} \leq T\right]} v\left(t_{n}, y\left(t_{n}\right)\right)\right], \\
v\left(t^{\prime}, x^{\prime}\right) & =\inf _{\varphi \in \tilde{D}_{a}} \sup _{\tau \in \Omega}\left[\int_{t^{\prime}}^{t_{n}} \psi\left(s, y^{\prime}(s), \tau(s)\right) \mathrm{d} s+\sum_{1 \leq k<n} C\left(t_{k}, y^{\prime}\left(t_{k}\right), \xi_{k}\right) \mathbb{1}_{\left[t_{k} \leq T\right]}+\mathbb{1}_{\left[t_{n} \leq T\right]} v\left(t_{n}, y^{\prime}\left(t_{n}\right)\right)\right] .
\end{aligned}
$$

Fix an arbitrary $\epsilon^{1}>0$. Let $\varphi=\left(\left(t_{n}\right)_{n \geq 1},\left(\xi_{n}\right)_{n \geq 1}\right)$ belongs to $\tilde{D}_{a}$ such that

$$
\begin{aligned}
& \sup _{\tau \in \Omega}\left[\int_{t}^{t_{n}} \psi(s, y(s), \tau(s)) \mathrm{d} s+\sum_{1 \leq k<n} C\left(t_{k}, y\left(t_{k}\right), \xi_{k}\right) \mathbb{1}_{\left[t_{k} \leq T\right]}+\mathbb{1}_{\left[t_{n} \leq T\right]} v\left(t_{n}, y\left(t_{n}\right)\right)\right] \\
& \leq \inf _{\varphi \in \tilde{D}_{a}} \sup _{\tau \in \Omega}\left[\int_{t}^{t_{n}} \psi(s, y(s), \tau(s)) \mathrm{d} s+\sum_{1 \leq k<n} C\left(t_{k}, y\left(t_{k}\right), \xi_{k}\right) \mathbb{1}_{\left[t_{k} \leq T\right]}+\mathbb{1}_{\left[t_{n} \leq T\right]} v\left(t_{n}, y\left(t_{n}\right)\right)\right]+\epsilon^{1} \\
& =v(t, x)+\epsilon^{1} .
\end{aligned}
$$

Also,

$$
v\left(t^{\prime}, x^{\prime}\right) \leq \sup _{\tau \in \Omega}\left[\int_{t^{\prime}}^{t_{n}} \psi\left(s, y^{\prime}(s), \tau(s)\right) \mathbb{1}_{\left[s \geq t^{\prime}\right]} \mathrm{d} s+\sum_{1 \leq k<n} C\left(t_{k}, y^{\prime}\left(t_{k}\right), \xi_{k}\right) \mathbb{1}_{\left[t_{k} \leq T\right]}+\mathbb{1}_{\left[t_{n} \leq T\right]} v\left(t_{n}, y^{\prime}\left(t_{n}\right)\right)\right] .
$$

Now pick $\tau_{1}$ such that

$$
\begin{aligned}
& \sup _{\tau \in \Omega}\left[\int_{t^{\prime}}^{t_{n}} \psi\left(s, y^{\prime}(s), \tau(s)\right) \mathbb{1}_{\left[s \geq t^{\prime}\right]} \mathrm{d} s+\sum_{1 \leq k<n} C\left(t_{k}, y^{\prime}\left(t_{k}\right), \xi_{k}\right) \mathbb{1}_{\left[t_{k} \leq T\right]}+\mathbb{1}_{\left[t_{n} \leq T\right]} v\left(t_{n}, y^{\prime}\left(t_{n}\right)\right)\right] \\
& \leq \int_{t^{\prime}}^{t_{n}} \psi\left(s, y^{\prime}(s), \tau^{1}(s)\right) \mathbb{1}_{\left[s \geq t^{\prime}\right]} \mathrm{d} s+\sum_{1 \leq k<n} C\left(t_{k}, y^{\prime}\left(t_{k}\right), \xi_{k}\right) \mathbb{1}_{\left[t_{k} \leq T\right]}+\mathbb{1}_{\left[t_{n} \leq T\right]} v\left(t_{n}, y^{\prime}\left(t_{n}\right)\right)+\epsilon^{1} .
\end{aligned}
$$


Then

$$
\begin{aligned}
v\left(t^{\prime}, x^{\prime}\right)-v(t, x) \leq & \int_{t^{\prime}}^{t_{n}} \psi\left(s, y^{\prime}(s), \tau^{1}(s)\right) \mathbb{1}_{\left[s \geq t^{\prime}\right]} \mathrm{d} s+\sum_{1 \leq k<n} C\left(t_{k}, y^{\prime}\left(t_{k}\right), \xi_{k}\right) \mathbb{1}_{\left[t_{k} \leq T\right]} \\
& \left.+\mathbb{1}_{\left[t_{n} \leq T\right]} v\left(t_{n}, y^{\prime}\left(t_{n}\right)\right)\right]-\int_{t}^{t_{n}} \psi\left(s, y(s), \tau^{1}(s)\right) \mathrm{d} s \\
& -\sum_{1 \leq k<n} C\left(t_{k}, y\left(t_{k}\right), \xi_{k}\right) \mathbb{1}_{\left[t_{k} \leq T\right]}-\mathbb{1}_{\left[t_{n} \leq T\right]} v\left(t_{n}, y\left(t_{n}\right)\right)+2 \epsilon^{1} .
\end{aligned}
$$

Next w.l.o.g we assume that $t^{\prime}<t$. Then we deduce that:

$$
\begin{aligned}
v\left(t^{\prime}, x^{\prime}\right)-v(t, x) \leq & \int_{t_{0}}^{t_{n}}\left\{\left(\psi\left(s, y^{\prime}(s), \tau^{1}(s)\right)-\psi\left(s, y(s), \tau^{1}(s)\right)\right) \mathbb{1}_{[s \geq t]}\right\} \mathrm{d} s+\int_{t_{0}}^{t_{n}} \psi\left(s, y^{\prime}(s), \tau^{1}(s)\right) \mathbb{1}_{\left[t^{\prime} \leq s<t\right]} \mathrm{d} s \\
& +\sum_{1 \leq k<n}\left\{C\left(t_{k}, y^{\prime}\left(t_{k}\right), \xi_{k}\right)-C\left(t_{k}, y\left(t_{k}\right), \xi_{k}\right)\right\} \mathbb{1}_{\left[t_{k} \leq T\right]} \\
& +\mathbb{1}_{\left[t_{n} \leq T\right]}\left\{v\left(t_{n}, y^{\prime}\left(t_{n}\right)\right)-v\left(t_{n}, y\left(t_{n}\right)\right)\right\}+2 \epsilon^{1} \\
\leq & \int_{t_{0}}^{t_{n}}\left\{\left|\psi\left(s, y^{\prime}(s), \tau^{1}(s)\right)-\psi\left(s, y(s), \tau^{1}(s)\right)\right| \mathbb{1}_{[s \geq t]}\right\}+\int_{t_{0}}^{t_{n}}\left|\psi\left(s, y^{\prime}(s), \tau^{1}(s)\right)\right| \mathbb{1}_{\left[t^{\prime} \leq s<t\right]} \mathrm{d} s \\
& +n \max _{1 \leq k \leq n}\left|C\left(t_{k}, y^{\prime}\left(t_{k}\right), \xi_{k}\right)-C\left(t_{k}, y\left(t_{k}\right), \xi_{k}\right)\right|+\mathbb{1}_{\left[t_{n} \leq T\right]}\left\{\left|v\left(t_{n}, y^{\prime}\left(t_{n}\right)\right)\right|+\left|v\left(t_{n}, y\left(t_{n}\right)\right)\right|\right\}+2 \epsilon^{1} .
\end{aligned}
$$

Using the uniform continuity of $\psi, C$ in $y$ and property (3.1), then the right-hand side of (3.4), the first and the second term converges to 0 as $t^{\prime}$ tends to $t$ and $x^{\prime}$ tends to $x$.

Now let us focus on the last one. Since $(\delta, \xi) \in \tilde{D}_{a}$ then

$$
\mathbb{1}_{\left[t_{n} \leq T\right]}\left\{\left|v\left(t_{n}, y^{\prime}\left(t_{n}\right)\right)\right|+\left|v\left(t_{n}, y\left(t_{n}\right)\right)\right|\right\} \leq C \frac{\left(1+|x|^{2}+\left|x^{\prime}\right|^{2}\right)}{n},
$$

where $C$ is a constant which comes from the linear growth of $\psi$ and $G$. Taking the limit as $\left(t^{\prime}, x^{\prime}\right) \rightarrow(t, x)$ we obtain:

$$
\limsup _{\left(t^{\prime}, x^{\prime}\right) \rightarrow(t, x)} v\left(t^{\prime}, x^{\prime}\right) \leq v(t, x)+C \frac{\left(1+|x|^{2}+\left|x^{\prime}\right|^{2}\right)}{n}+2 \epsilon^{1} .
$$

As $n$ and $\epsilon^{1}$ are arbitrary then sending $n \rightarrow+\infty$ and $\epsilon^{1} \rightarrow 0$, to obtain:

$$
\limsup _{\left(t^{\prime}, x^{\prime}\right) \rightarrow(t, x)} v\left(t^{\prime}, x^{\prime}\right) \leq v(t, x) .
$$

Therefore $v$ is upper semi-continuous.

Now we show that $v$ is lower semi-continuous.

Fix an arbitrary $\epsilon_{2}>0$. Let $\varphi_{2}=\left(\left(t_{n}\right)_{n \geq 1},\left(\xi_{n}\right)_{n \geq 1}\right)$ belongs to $\tilde{D}_{a}$ such that

$$
\begin{aligned}
& \sup _{\tau \in \Omega}\left[\int_{t^{\prime}}^{t_{n}} \psi\left(s, y^{\prime}(s), \tau(s)\right) \mathrm{d} s+\sum_{1 \leq k<n} C\left(t_{k}, y^{\prime}\left(t_{k}\right), \xi_{k}\right) \mathbb{1}_{\left[t_{k} \leq T\right]}+\mathbb{1}_{\left[t_{n} \leq T\right]} v\left(t_{n}, y^{\prime}\left(t_{n}\right)\right)\right] \\
& \leq \inf _{\varphi_{2} \in \tilde{D}_{a}} \sup _{\tau \in \Omega}\left[\int_{t}^{t_{n}} \psi\left(s, y^{\prime}(s), \tau(s)\right) \mathrm{d} s+\sum_{1 \leq k<n} C\left(t_{k}, y^{\prime}\left(t_{k}\right), \xi_{k}\right) \mathbb{1}_{\left[t_{k} \leq T\right]}+\mathbb{1}_{\left[t_{n} \leq T\right]} v\left(t_{n}, y\left(t_{n}\right)\right)\right]+\epsilon_{2} \\
& =v\left(t^{\prime}, x^{\prime}\right)+\epsilon_{2} .
\end{aligned}
$$


Also,

$$
v(t, x) \leq \sup _{\tau \in \Omega}\left[\int_{t}^{t_{n}} \psi(s, y(s), \tau(s)) \mathbb{1}_{[s \geq t]} \mathrm{d} s+\sum_{1 \leq k<n} C\left(t_{k}, y\left(t_{k}\right), \xi_{k}\right) \mathbb{1}_{\left[t_{k} \leq T\right]}+\mathbb{1}_{\left[t_{n} \leq T\right]} v\left(t_{n}, y\left(t_{n}\right)\right)\right],
$$

now, pick $\tau_{2}$ such that

$$
\begin{aligned}
& \sup _{\tau \in \Omega} {\left[\int_{t}^{t_{n}} \psi(s, y(s), \tau(s)) \mathbb{1}_{[s \geq t]} \mathrm{d} s+\sum_{1 \leq k<n} C\left(t_{k}, y\left(t_{k}\right), \xi_{k}\right) \mathbb{1}_{\left[t_{k} \leq T\right]}+\mathbb{1}_{\left[t_{n} \leq T\right]} v\left(t_{n}, y\left(t_{n}\right)\right)\right] } \\
& \leq \int_{t}^{t_{n}} \psi\left(s, y(s), \tau_{2}(s)\right) \mathbb{1}_{[s \geq t]} \mathrm{d} s+\sum_{1 \leq k<n} C\left(t_{k}, y\left(t_{k}\right), \xi_{k}\right) \mathbb{1}_{\left[t_{k} \leq T\right]}+\mathbb{1}_{\left[t_{n} \leq T\right]} v\left(t_{n}, y\left(t_{n}\right)\right)+\epsilon_{2} .
\end{aligned}
$$

Then

$$
\begin{aligned}
v\left(t^{\prime}, x^{\prime}\right)-v(t, x) \geq & \int_{t^{\prime}}^{t_{n}} \psi\left(s, y^{\prime}(s), \tau_{2}(s)\right) \mathrm{d} s+\sum_{1 \leq k<n} C\left(t_{k}, y^{\prime}\left(t_{k}\right), \xi_{k}\right) \mathbb{1}_{\left[t_{k} \leq T\right]} \\
& \left.+\mathbb{1}_{\left[t_{n} \leq T\right]} v\left(t_{n}, y^{\prime}\left(t_{n}\right)\right)\right]-\int_{t}^{t_{n}} \psi\left(s, y(s), \tau_{2}(s)\right) \mathrm{d} s-\mathbb{1}_{\left[t_{n} \leq T\right]} v\left(t_{n}, y\left(t_{n}\right)\right) \\
& -\sum_{1 \leq k<n} C\left(t_{k}, y\left(t_{k}\right), \xi_{k}\right) \mathbb{1}_{\left[t_{k} \leq T\right]}-2 \epsilon_{2} .
\end{aligned}
$$

Next w.l.o.g we assume that $t^{\prime}<t$. Then we deduce that:

$$
\begin{aligned}
v\left(t^{\prime}, x^{\prime}\right)-v(t, x) \geq & \int_{t_{0}}^{t_{n}}\left\{\left(\psi\left(s, y^{\prime}(s), \tau_{2}(s)\right)-\psi\left(s, y(s), \tau_{2}(s)\right)\right) \mathbb{1}_{[s \geq t]}\right\} \mathrm{d} s+\int_{t_{0}}^{t_{n}} \psi\left(s, y^{\prime}(s), \tau_{2}(s)\right) \mathbb{1}_{\left[t^{\prime} \leq s<t\right]} \mathrm{d} s \\
& +\sum_{1 \leq k<n}\left\{C\left(t_{k}, y^{\prime}\left(t_{k}\right), \xi_{k}\right)-C\left(t_{k}, y\left(t_{k}\right), \xi_{k}\right)\right\} \mathbb{1}_{\left[t_{k} \leq T\right]} \\
& +\mathbb{1}_{\left[t_{n} \leq T\right]}\left\{v\left(t_{n}, y^{\prime}\left(t_{n}\right)\right)-v\left(t_{n}, y\left(t_{n}\right)\right)\right\}-2 \epsilon_{2} \\
\geq & -\int_{t_{0}}^{t_{n}}\left\{\left|\psi\left(s, y^{\prime}(s), \tau_{2}(s)\right)-\psi\left(s, y(s), \tau_{2}(s)\right)\right| \mathbb{1}_{[s \geq t]}\right\} \mathrm{d} s \\
& -\int_{t_{0}}^{t_{n}}\left|\psi\left(s, y^{\prime}(s), \tau_{2}(s)\right)\right| \mathbb{1}_{\left[t^{\prime} \leq s<t\right]} \mathrm{d} s-n \max _{1 \leq k \leq n}\left|C\left(t_{k}, y^{\prime}\left(t_{k}\right), \xi_{k}\right)-C\left(t_{k}, y\left(t_{k}\right), \xi_{k}\right)\right| \\
& -\mathbb{1}_{\left[t_{n} \leq T\right]}\left\{\left|v\left(t_{n}, y^{\prime}\left(t_{n}\right)\right)\right|+\left|v\left(t_{n}, y\left(t_{n}\right)\right)\right|\right\}-2 \epsilon_{2} .
\end{aligned}
$$

Using the uniform continuity of $\psi, C$ in $y$ and property (3.1). Then the right-hand side of (3.4) the first and the second term converges to 0 as $t^{\prime} \rightarrow t$ and $x^{\prime} \rightarrow x$.

Now let us focus on the last one. Since $\left(\left(t_{n}\right)_{n \geq 1},\left(\xi_{n}\right)_{n \geq 1}\right)$ be a admissible control then

$$
-\mathbb{1}_{\left[t_{n} \leq T\right]}\left\{\left|v\left(t_{n}, y^{\prime}\left(t_{n}\right)\right)\right|+\left|v\left(t_{n}, y\left(t_{n}\right)\right)\right|\right\} \geq-C \frac{\left(1+|x|^{2}+\left|x^{\prime}\right|^{2}\right)}{n},
$$

where $C$ is a constant which come from the linear growth of $\psi$ and $G$, taking the limit as $\left(t^{\prime}, x^{\prime}\right) \rightarrow(t, x)$ to obtain:

$$
\liminf _{\left(t^{\prime}, x^{\prime}\right) \rightarrow(t, x)} v\left(t^{\prime}, x^{\prime}\right) \geq v(t, x)-C \frac{\left(1+|x|^{2}+\left|x^{\prime}\right|^{2}\right)}{n}-2 \epsilon_{2} .
$$


As $n$ and $\epsilon_{2}$ are arbitraries then putting $n \rightarrow+\infty$ and $\epsilon_{2} \rightarrow 0$ to obtain:

$$
\liminf _{\left(t^{\prime}, x^{\prime}\right) \rightarrow(t, x)} v\left(t^{\prime}, x^{\prime}\right) \geq v(t, x) .
$$

Therefore $v$ is lower semi-continuous. We then proved that $v$ is continuous.

\subsection{Terminal value}

Because of the possible jumps at the terminal time $T$, it is easy to see that, in general, $v(t, x)$ does not tend to $G(x)$ as t tends to $T$. Extend the set of jumps to include jumps of zero, meaning no jump. Call this extended set $E_{0}$, extend trivially the operator $N$ to a function independent from $t$, and let

$$
G_{1}(x)=\inf _{\xi \in E_{0}}[G(x+g(T, x, \xi))+C(T, x, \xi)]=\min \{G(x), N[G](T, x)\} .
$$

We know that $G$ and $C$ are uniformly continuous in $x$ then $G_{1}(x)$ is continuous. We claim

\section{Lemma 3.5.}

$$
v(t, x) \rightarrow G_{1}(x) \quad \text { as } \quad t \rightarrow T .
$$

Proof. Fix $(t, x)$ and a strategy $\varphi$. As in the previous proof, for each $\tau(\Delta)$, gather all jumps of $\varphi(\tau)$ if any, in jump $\xi_{1}$ at the time $T$. Then we have

$$
\left|J(t, x, \varphi, \tau)-G\left(x+g\left(T, x, \xi_{1}\right)\right)-C\left(T, x, \xi_{1}\right)\right| \leq C_{x}(T-t)
$$

or

$$
J(t, x, \varphi, \tau)=G\left(x+g\left(T, x, \xi_{1}\right)\right)+C\left(T, x, \xi_{1}\right)+O(T-t) .
$$

The right hand side above only depends on $\xi_{1}$, not on $\tau($.) itself. It follows that

$$
\begin{aligned}
\inf _{\varphi} \sup _{\tau} J(t, x, \varphi, \tau) & =\inf _{\xi \in E_{0}}[G(x+g(T, x, \xi))+C(T, x, \xi)]+O(T-t) \\
& =G_{1}(x)+O(T-t) .
\end{aligned}
$$

The result follows letting $t \rightarrow T$.

\section{Viscosity CHARACTERIZATION OF THE VALUE FUNCTION}

In this section we prove that the value function $v$ is a viscosity solution of the Hamilton-Jacobi-Isaacs quasivariational inequality, that we replace by an equivalent QVI easier to investigate.

We now consider the following quasi-variational inequality (Isaacs equation):

$$
\max \left\{\min _{\tau \in \mathcal{K}}\left[-\frac{\partial v}{\partial t}-\frac{\partial v}{\partial x} f(t, x, \tau)-\psi(t, x, \tau)\right], v(t, x)-N[v](t, x)\right\}=0,
$$

with the terminal condition: $v(t, x)=G_{1}(x), x \in \mathbb{R}^{m}$, where $G_{1}$ is given by (3.6).

Notice that it follows from hypothesis that the term in square brackets in (4.1) above is continuous with respect to $\tau$ so that the minimum in $\tau$ over the compact $\mathcal{K}$ exists.

Recall the notion of viscosity solution of QVI (4.1). 
Definition 4.1. Let $v$ be a continuous function defined on $[0, T] \times \mathbb{R}^{m}, \mathbb{R}$-valued and such that $v(T, x)=G_{1}(x)$ for any $x \in \mathbb{R}^{m}$. The $v$ is called:

(i) A viscosity supersolution of (4.1) if for any $(\bar{t}, \bar{x}) \in\left[t_{0}, T\left[\times \mathbb{R}^{m}\right.\right.$ and any function $\varphi \in C^{1,2}\left(\left[t_{0}, T\left[\times \mathbb{R}^{m}\right)\right.\right.$ such that $\varphi(\bar{t}, \bar{x})=v(\bar{t}, \bar{x})$ and $(\bar{t}, \bar{x})$ is a local maximum of $\varphi-v$, we have:

$$
\max \left\{\min _{\tau \in \mathcal{K}}\left[-\frac{\partial \varphi}{\partial t}-\frac{\partial \varphi}{\partial x} f(\bar{t}, \bar{x}, \tau)-\psi(\bar{t}, \bar{x}, \tau)\right], v(\bar{t}, \bar{x})-N[v](\bar{t}, \bar{x})\right\} \geq 0 ;
$$

(ii) a viscosity subsolution of (4.1) if for any $(\bar{t}, \bar{x}) \in\left[t_{0}, T\left[\times \mathbb{R}^{m}\right.\right.$ and any function $\varphi \in C^{1,2}\left(\left[t_{0}, T\left[\times \mathbb{R}^{m}\right)\right.\right.$ such that $\varphi(\bar{t}, \bar{x})=v(\bar{t}, \bar{x})$ and $(\bar{t}, \bar{x})$ is a local minimum of $\varphi-v$, we have:

$$
\max \left\{\min _{\tau \in \mathcal{K}}\left[-\frac{\partial \varphi}{\partial t}-\frac{\partial \varphi}{\partial x} f(\bar{t}, \bar{x}, \tau)-\psi(\bar{t}, \bar{x}, \tau)\right], v(\bar{t}, \bar{x})-N[v](\bar{t}, \bar{x})\right\} \leq 0 ;
$$

(iii) a viscosity solution if it is both a viscosity supersolution and subsolution.

Theorem 4.2. The value function $v$ is the viscosity solution of the quasi-variational inequality (4.1).

Proof. The viscosity property follows from the dynamic programming principle and is proved in [10].

Now we give an equivalent of quasi-variational inequality (4.1). In this section, we consider the new function $\Gamma$ given by the classical change of variable $\Gamma(t, x)=\exp (t) v(t, x)$, for any $t \in\left[t_{0}, T\right]$ and $x \in \mathbb{R}^{m}$. Of course, the function $\Gamma$ is bounded from below and continuous with respect to its arguments.

A second property is given by the:

Proposition 4.3. $v$ is a viscosity solution of (4.1) if and only if $\Gamma$ is a viscosity solution to the following quasi-variational inequality in $\left[t_{0}, T\left[\times \mathbb{R}^{m}\right.\right.$,

$$
\max \left\{\min _{\tau}\left[-\frac{\partial \Gamma}{\partial t}+\Gamma(t, x)-\frac{\partial \Gamma}{\partial x} f(t, x, \tau)-\exp (t) \psi(t, x, \tau)\right], \Gamma(t, x)-M[\Gamma](t, x)\right\}=0,
$$

where $M[\Gamma](t, x)=\inf _{\xi \in E}[\Gamma(t, x+g(t, x, \xi))+\exp (t) C(t, x, \xi)]$. The terminal condition for $\Gamma$ is: $\Gamma(T, x)=$ $\exp (T) G_{1}(x)$ in $\mathbb{R}^{m}$.

\section{UNIQUENESS OF THE SOLUTION OF QUASI-VARIATIONAL INEQUALITY}

We are going now to address the question of uniqueness of the viscosity solution of quasi-variational inequality (4.1). We have the following:

Theorem 5.1. The solution in viscosity sense of quasi-variational inequality (4.1) is unique in the space of continuous functions on $\left[t_{0}, T\right] \times R^{m}$ which satisfy a linear growth condition, i.e., in the space

$$
\begin{aligned}
& \mathcal{C}:=\left\{\varphi:[0, T] \times \mathbb{R}^{m} \rightarrow \mathbb{R},\right. \text { continuous and for any } \\
& \qquad(t, x), \varphi(t, x) \leq C(1+|x|) \text { for some constants } C \text { and bounded from below }\} .
\end{aligned}
$$

Proof. We will show by contradiction that if $u$ and $w$ is a subsolution and a supersolution respectively for (4.4) then $u \leq w$. Therefore if we have two solutions of (4.4) then they are obviously equal. Actually for some $R>0$ suppose there exists $(\bar{t}, \bar{x}) \in\left[t_{0}, T\right] \times B_{R} \times \mathcal{I}\left(B_{R}:=\left\{x \in \mathbb{R}^{m} ;|x|<R\right\}\right)$ such that:

$$
\max _{t, x}(u(t, x)-w(t, x))=u(\bar{t}, \bar{x})-w(\bar{t}, \bar{x})=\eta>0 .
$$


Let us take $\theta, \lambda$ and $\beta \in(0,1]$ small enough. Then, for a small $\epsilon>0$, let us define:

$$
\Phi_{\epsilon}(t, x, y)=(1-\lambda) u(t, x)-w(t, y)-\frac{1}{2 \epsilon}|x-y|^{2}-\theta\left(|x-\bar{x}|^{4}+|y-\bar{x}|^{4}\right)-\beta(t-\bar{t})^{2} .
$$

By the linear growth assumption on $u$ and $w$, there exists a $\left(t_{\epsilon}, x_{\epsilon}, y_{\epsilon}\right) \in\left[t_{0}, T\right] \times B_{R} \times B_{R}$, for $R$ large enough, such that:

$$
\Phi_{\epsilon}\left(t_{\epsilon}, x_{\epsilon}, y_{\epsilon}\right)=\max _{(t, x, y)} \Phi_{\epsilon}(t, x, y) .
$$

On the other hand, from $2 \Phi_{\epsilon}\left(t_{\epsilon}, x_{\epsilon}, y_{\epsilon}\right) \geq \Phi_{\epsilon}\left(t_{\epsilon}, x_{\epsilon}, x_{\epsilon}\right)+\Phi_{\epsilon}\left(t_{\epsilon}, y_{\epsilon}, y_{\epsilon}\right)$, we have

$$
\frac{1}{\epsilon}\left|x_{\epsilon}-y_{\epsilon}\right|^{2} \leq(1-\lambda)\left(u\left(t_{\epsilon}, x_{\epsilon}\right)-u\left(t_{\epsilon}, y_{\epsilon}\right)\right)+\left(w\left(t_{\epsilon}, x_{\epsilon}\right)-w\left(t_{\epsilon}, y_{\epsilon}\right)\right),
$$

and consequently $\frac{1}{\epsilon}\left|x_{\epsilon}-y_{\epsilon}\right|^{2}$ is bounded, and as $\epsilon \rightarrow 0,\left|x_{\epsilon}-y_{\epsilon}\right| \rightarrow 0$. Since $u$ and $w$ are uniformly continuous on $[0, T] \times \bar{B}_{R}$, then $\frac{1}{2 \epsilon}\left|x_{\epsilon}-y_{\epsilon}\right|^{2} \rightarrow 0$ as $\epsilon \rightarrow 0$.

Since

$$
(1-\lambda) u(\bar{t}, \bar{x})-w(\bar{t}, \bar{x}) \leq \Phi_{\epsilon}\left(t_{\epsilon}, x_{\epsilon}, y_{\epsilon}\right) \leq(1-\lambda) u\left(t_{\epsilon}, x_{\epsilon}\right)-w\left(t_{\epsilon}, y_{\epsilon}\right),
$$

it follow as $\lambda \rightarrow 0$ and the continuity of $u$ and $w$ that, up to a subsequence,

$$
\left(t_{\epsilon}, x_{\epsilon}, y_{\epsilon}\right) \rightarrow(\bar{t}, \bar{x}, \bar{x})
$$

Next let us show that $t_{\epsilon}<T$. Actually if $t_{\epsilon}=T$ then,

$$
\Phi_{\epsilon}(\bar{t}, \bar{x}, \bar{x}) \leq \Phi_{\epsilon}\left(T, x_{\epsilon}, y_{\epsilon}\right)
$$

and,

$$
(1-\lambda) u(\bar{t}, \bar{x})-w(\bar{t}, \bar{x}) \leq(1-\lambda) \exp (T) G_{1}\left(x_{\epsilon}\right)-\exp (T) G_{1}\left(y_{\epsilon}\right)-\beta\left(T-t_{\epsilon}\right)^{2},
$$

since $u\left(T, x_{\epsilon}\right)=\exp (T) G_{1}\left(x_{\epsilon}\right), w\left(T, y_{\epsilon}\right)=\exp (T) G_{1}\left(y_{\epsilon}\right)$ and $G_{1}$ is uniformly continuous on $\bar{B}_{R}$. Then as $\lambda \rightarrow 0$ we have,

$$
\begin{aligned}
& \eta \leq-\beta(T-\bar{t})^{2} \\
& \eta<0,
\end{aligned}
$$

which yields a contradiction and we have $t_{\epsilon} \in\left[t_{0}, T\right)$. We now claim that:

$$
w\left(t_{\epsilon}, y_{\epsilon}\right)-\inf _{\xi \in E}\left[w\left(t_{\epsilon}, y_{\epsilon}+g\left(t_{\epsilon}, y_{\epsilon}, \xi\right)\right)+\exp \left(t_{\epsilon}\right) C\left(t_{\epsilon}, y_{\epsilon}, \xi\right)\right]<0 .
$$

Indeed if

$$
w\left(t_{\epsilon}, y_{\epsilon}\right)-\inf _{\xi \in E}\left[w\left(t_{\epsilon}, y_{\epsilon}+g\left(t_{\epsilon}, y_{\epsilon}, \xi\right)\right)+\exp \left(t_{\epsilon}\right) C\left(t_{\epsilon}, y_{\epsilon}, \xi\right)\right] \geq 0,
$$

then from Proposition 3.2 we have:

$$
w\left(t_{\epsilon}, y_{\epsilon}\right)-\inf _{\xi \in E}\left[w\left(t_{\epsilon}, y_{\epsilon}+g\left(t_{\epsilon}, y_{\epsilon}, \xi\right)\right)+\exp \left(t_{\epsilon}\right) C\left(t_{\epsilon}, y_{\epsilon}, \xi\right)\right]=0,
$$

then there exist $\xi_{1} \in E$ and small $\epsilon_{1}>0$ such that:

$$
w\left(t_{\epsilon}, y_{\epsilon}\right)-w\left(t_{\epsilon}, y_{\epsilon}+g\left(t_{\epsilon}, y_{\epsilon}, \xi_{1}\right)\right)-\exp \left(t_{\epsilon}\right) C\left(t_{\epsilon}, y_{\epsilon}, \xi_{1}\right) \geq-\epsilon_{1} .
$$

From the subsolution property of $u\left(t_{\epsilon}, x_{\epsilon}\right)$, we have

$$
u\left(t_{\epsilon}, x_{\epsilon}\right)-\inf _{\xi \in E}\left[u\left(t_{\epsilon}, x_{\epsilon}+g\left(t_{\epsilon}, x_{\epsilon}, \xi\right)\right)+\exp \left(t_{\epsilon}\right) C\left(t_{\epsilon}, x_{\epsilon}, \xi\right)\right] \leq 0,
$$


then

$$
u\left(t_{\epsilon}, x_{\epsilon}\right)-u\left(t_{\epsilon}, x_{\epsilon}+g\left(t_{\epsilon}, x_{\epsilon}, \xi_{1}\right)\right)-\exp \left(t_{\epsilon}\right) C\left(t_{\epsilon}, x_{\epsilon}, \xi_{1}\right) \leq 0 .
$$

It follows that:

$$
\begin{aligned}
& (1-\lambda)\left(u\left(t_{\epsilon}, x_{\epsilon}\right)-w\left(t_{\epsilon}, y_{\epsilon}\right)-\left[(1-\lambda) u\left(t_{\epsilon}, x_{\epsilon}+g\left(t_{\epsilon}, x_{\epsilon}, \xi_{1}\right)\right)-w\left(t_{\epsilon}, y_{\epsilon}+g\left(t_{\epsilon}, y_{\epsilon}, \xi_{1}\right)\right)\right]\right. \\
& \quad \leq(1-\lambda) \exp \left(t_{\epsilon}\right) C\left(t_{\epsilon}, x_{\epsilon}, \xi_{1}\right)-\exp \left(t_{\epsilon}\right) C\left(t_{\epsilon}, y_{\epsilon}, \xi_{1}\right)+\epsilon_{1} .
\end{aligned}
$$

Now since $C \geq \alpha>0$, then

$$
\begin{aligned}
& (1-\lambda)\left(u\left(t_{\epsilon}, x_{\epsilon}\right)-w\left(t_{\epsilon}, y_{\epsilon}\right)-\left[(1-\lambda) u\left(t_{\epsilon}, x_{\epsilon}+g\left(t_{\epsilon}, x_{\epsilon}, \xi_{1}\right)\right)-w\left(t_{\epsilon}, y_{\epsilon}+g\left(t_{\epsilon}, y_{\epsilon}, \xi_{1}\right)\right)\right]\right. \\
& \quad<-\lambda \alpha+\exp \left(t_{\epsilon}\right) C\left(t_{\epsilon}, x_{\epsilon}, \xi_{1}\right)-\exp \left(t_{\epsilon}\right) C\left(t_{\epsilon}, y_{\epsilon}, \xi_{1}\right)+\epsilon_{1} .
\end{aligned}
$$

But this contradicts the definition of (5.1), since $C, u, w$ is uniformly continuous on $[0, T] \times \bar{B}_{R}$ and sending $\epsilon_{1} \rightarrow 0$ and the claim (5.5) holds.

Next let us denote

$$
\varphi_{\epsilon}(t, x, y)=\frac{1}{2 \epsilon}|x-y|^{2}+\theta\left(|x-\bar{x}|^{4}+|y-\bar{x}|^{4}\right)+\beta(t-\bar{t})^{2} .
$$

Then we have:

$$
\left\{\begin{array}{l}
D_{t} \varphi_{\epsilon}(t, x, y)=2 \beta(t-\bar{t}), \\
D_{x} \varphi_{\epsilon}(t, x, y)=\frac{1}{\epsilon}(x-y)+4 \theta(x-\bar{x})|x-\bar{x}|^{2}, \\
D_{y} \varphi_{\epsilon}(t, x, y)=-\frac{1}{\epsilon}(x-y)+4 \theta(y-\bar{x})|y-\bar{x}|^{2} .
\end{array}\right.
$$

Let $c, d \in \mathbb{R}$ such that

$$
c+d=2 \beta\left(t_{\epsilon}-\bar{t}\right) .
$$

Taking now into account (5.5), (5.8), and the definition of viscosity solution, we get:

$$
\min _{\tau}\left[-c+(1-\lambda) u\left(t_{\epsilon}, x_{\epsilon}\right)-\left\langle\frac{1}{\epsilon}\left(x_{\epsilon}-y_{\epsilon}\right)+4 \theta\left(x_{\epsilon}-\bar{x}\right)\left|x_{\epsilon}-\bar{x}\right|^{2}, f\left(t_{\epsilon}, x_{\epsilon}, \tau\right)\right\rangle-(1-\lambda) \exp \left(t_{\epsilon}\right) \psi\left(t_{\epsilon}, x_{\epsilon}, \tau\right)\right] \leq 0
$$

and

$$
\min _{\tau}\left[d+w\left(t_{\epsilon}, y_{\epsilon}\right)-\left\langle\frac{1}{\epsilon}\left(x_{\epsilon}-y_{\epsilon}\right)-4 \theta\left(y_{\epsilon}-\bar{x}\right)\left|y_{\epsilon}-\bar{x}\right|^{2}, f\left(t_{\epsilon}, y_{\epsilon}, \tau\right)\right\rangle-\exp \left(t_{\epsilon}\right) \psi\left(t_{\epsilon}, y_{\epsilon}, \tau\right)\right] \geq 0,
$$

which implies that:

$$
\begin{aligned}
-c-d+(1-\lambda) u\left(t_{\epsilon}, x_{\epsilon}\right)-w\left(t_{\epsilon}, y_{\epsilon}\right) & \\
\leq & \min _{\tau}\left[-\left\langle\frac{1}{\epsilon}\left(x_{\epsilon}-y_{\epsilon}\right)-4 \theta\left(y_{\epsilon}-\bar{x}\right)\left|y_{\epsilon}-\bar{x}\right|^{2}, f\left(t_{\epsilon}, y_{\epsilon}, \tau\right)\right\rangle-\exp \left(t_{\epsilon}\right) \psi\left(t_{\epsilon}, y_{\epsilon}, \tau\right)\right] \\
& -\min _{\tau}\left[-\left\langle\frac{1}{\epsilon}\left(x_{\epsilon}-y_{\epsilon}\right)+4 \theta\left(x_{\epsilon}-\bar{x}\right)\left|x_{\epsilon}-\bar{x}\right|^{2}, f\left(t_{\epsilon}, x_{\epsilon}, \tau\right)\right\rangle-(1-\lambda) \exp \left(t_{\epsilon}\right) \psi\left(t_{\epsilon}, x_{\epsilon}, \tau\right)\right], \\
\leq & \sup _{\tau}\left[\left\langle\frac{1}{\epsilon}\left(x_{\epsilon}-y_{\epsilon}\right)+4 \theta\left(x_{\epsilon}-\bar{x}\right)\left|x_{\epsilon}-\bar{x}\right|^{2}, f\left(t_{\epsilon}, x_{\epsilon}, \tau\right)\right\rangle+(1-\lambda) \exp \left(t_{\epsilon}\right) \psi\left(t_{\epsilon}, x_{\epsilon}, \tau\right)\right] \\
& -\sup _{\tau}\left[\left\langle\frac{1}{\epsilon}\left(x_{\epsilon}-y_{\epsilon}\right)-4 \theta\left(y_{\epsilon}-\bar{x}\right)\left|y_{\epsilon}-\bar{x}\right|^{2}, f\left(t_{\epsilon}, y_{\epsilon}, \tau\right)\right\rangle+\exp \left(t_{\epsilon}\right) \psi\left(t_{\epsilon}, y_{\epsilon}, \tau\right)\right], \\
\leq & \sup _{\tau}\left[\left\langle\frac{1}{\epsilon}\left(x_{\epsilon}-y_{\epsilon}\right), f\left(t_{\epsilon}, x_{\epsilon}, \tau\right)-f\left(t_{\epsilon}, y_{\epsilon}, \tau\right)\right\rangle\right. \\
& +\left\langle 4 \theta\left(x_{\epsilon}-\bar{x}\right)\left|x_{\epsilon}-\bar{x}\right|^{2}, f\left(t_{\epsilon}, x_{\epsilon}, \tau\right)\right\rangle+\left\langle 4 \theta\left(y_{\epsilon}-\bar{x}\right)\left|y_{\epsilon}-\bar{x}\right|^{2}, f\left(t_{\epsilon}, y_{\epsilon}, \tau\right)\right\rangle \\
& \left.+(1-\lambda) \exp \left(t_{\epsilon}\right) \psi\left(t_{\epsilon}, x_{\epsilon}, \tau\right)-\exp \left(t_{\epsilon}\right) \psi\left(t_{\epsilon}, y_{\epsilon}, \tau\right)\right] .
\end{aligned}
$$


Now, from (2.3), we get:

$$
\left\langle\frac{1}{\epsilon}\left(x_{\epsilon}-y_{\epsilon}\right), f\left(t_{\epsilon}, x_{\epsilon}, \tau\right)-f\left(t_{\epsilon}, y_{\epsilon}, \tau\right)\right\rangle \leq \frac{C}{\epsilon}\left|x_{\epsilon}-y_{\epsilon}\right|^{2} .
$$

Next

and finally,

$$
\left\langle 4 \theta\left(x_{\epsilon}-\bar{x}\right)\left|x_{\epsilon}-\bar{x}\right|^{2}, f\left(t_{\epsilon}, x_{\epsilon}, \tau\right)\right\rangle \leq 4 C \theta\left|x_{\epsilon}\right|\left|x_{\epsilon}-\bar{x}\right|^{3},
$$

Taking in to account

$$
\left\langle 4 \theta\left(y_{\epsilon}-\bar{x}\right)\left|y_{\epsilon}-\bar{x}\right|^{2}, f\left(t_{\epsilon}, y_{\epsilon}, \tau\right)\right\rangle \leq 4 C \theta\left|y_{\epsilon}\right|\left|y_{\epsilon}-\bar{x}\right|^{3} .
$$

$$
c+d=2 \beta\left(t_{\epsilon}-\bar{t}\right) .
$$

So that by plugging into (5.12) and note that $\lambda>0$ we obtain:

$$
\begin{aligned}
-2 \beta\left(t_{\epsilon}-\bar{t}\right)+(1-\lambda) u\left(t_{\epsilon}, x_{\epsilon}\right)-w\left(t_{\epsilon}, y_{\epsilon}\right) \leq & \frac{C}{\epsilon}\left|x_{\epsilon}-y_{\epsilon}\right|^{2}+4 C \theta\left|x_{\epsilon}\right|\left|x_{\epsilon}-\bar{x}\right|^{3}+4 C \theta\left|y_{\epsilon}\right|\left|y_{\epsilon}-\bar{x}\right|^{3} \\
& \left.+\sup _{\tau}(1-\lambda) \exp \left(t_{\epsilon}\right) \psi\left(t_{\epsilon}, x_{\epsilon}, \tau\right)-\exp \left(t_{\epsilon}\right) \psi\left(t_{\epsilon}, y_{\epsilon}, \tau\right)\right] .
\end{aligned}
$$

By sending $\epsilon \rightarrow 0, \lambda \rightarrow 0, \theta \rightarrow 0$ and taking into account of the continuity of $\psi$, we obtain $\eta \leq 0$ which is a contradiction. The proof of Theorem 5.1 is now complete.

As an example of a use of this result in mathematical finance, one may consider the option pricing problem of references $[4,5]$. If the piecewise linear transaction costs are replaced by a more realistic piecewise affine cost, i.e. a fixed cost is charged for any transaction in addition to a variable part, then the problem at hand is exactly that considered here.

Acknowledgements. The author thanks gratefully Prof. A. Popier for the fructuous discussions during the preparation of this paper. I also would like to thank the referees for their careful reading and for their helpful comments and suggestions that led to considerable improvements in the paper.

\section{REFERENCES}

[1] G. Barles, Deterministic impulse control problems. SIAM J. Control Optim. 23 (1985) 419-432.

[2] E.N. Barron, L.C. Evans and R. Jensen, Viscosity solutions of Isaaes' equations and differential games with Lipschitz controls. J. Differential Equations 53 (1984) 213-233.

[3] A. Bensoussan and J.L. Lions, Impulse Control and Quasi-Variational Inequalities. Bordes, Paris (1984)

[4] P. Bernhard, A robust control approach to option pricing including transaction costs. Annals of International Society of Dynamic Games, Birkäuser, Boston 7 (2005) 391-416.

[5] P. Bernhard, N. El Farouq and S. Thiery, An impulsive differential game arising in finance with interesting singularities. Annals of International Society of Dynamic Games, Birkäuser, Boston 8 (2006) 335-363.

[6] M. Crandall, H. Ishii and P.L. Lions, Users guide to viscosity solutions of second order partial differential equations, Bull. Amer. Math. Soc. 27 (1992) 1-67.

[7] S. Dharmatti and M. Ramaswamy, Zero-sum differential games involving hybrid controls. J. Optim. Theory Appl. 128 (2006) 75-102.

[8] S. Dharmatti and A.J. Shaiju, Infinite dimensional differential games with hybrid controls. Proc. Indian Acad. Sci. Math. 117 (2007) 233-257.

[9] B. El Asri, Optimal multi-modes switching problem in infinite horizon. Stoc. Dyn. 10 (2010) 231-261.

[10] N. El Farouq, G. Barles and P. Bernhard, Deterministic minimax impulse control. Appl. Math. Optim. (2010) DOI: 10.1007/s00245-009-9090-0.

[11] L.C. Evans and P.E. Souganidis, Differential games and representation formulas for the solution of Hamilton-Jacobi-Isaacs equations. Indiana Univ. J. Math. 33 (1984) 773-797.

[12] W.H. Fleming, The convergence problem for differential games. Ann. Math. Study 52 (1964) 195-210.

[13] P.L. Lions, Generalized Solutions of Hamilton-Jacobi Equations. Pitman, London (1982)

[14] P.L. Lions and P.E. Souganidis, Differential games, optimal control and directional derivatives of viscosity solutions of Bellmans and Isaacs equations. SIAM J. Control Optim. 23 (1985) 566-583. 
[15] A.J. Shaiju and S. Dharmatti, Differential games with continuous, switching and impulse controls. Nonlinear Anal. 63 (2005) 23-41.

[16] P.E. Souganidis, Max-min representations and product formulas for the viscosity solutions of Hamilton-Jacobi equations with applications to differential games. Nonlinear Anal. 9 (1985) 217-57.

[17] J.M. Yong, Systems governed by ordinary differential equations with continuous, switching and impulse controls. Appl. Math. Optim. 20 (1989) 223-235.

[18] J.M. Yong, Optimal switching and impulse controls for distributed parameter systems. Systems Sci. Math. Sci. 2 (1989) 137-160.

[19] J.M. Yong, Differential games with switching strategies. J. Math. Anal. Appl. 145 (1990) 455-469.

[20] J.M. Yong, A zero-sum differential game in a finite duration with switching strategies. SIAM J. Control Optim. 28 (1990) 1234-1250.

[21] J.M. Yong, Zero-sum differential games involving impulse controls. Appl. Math. Optim. 29 (1994) $243-261$. 